Bull. Mater. Sci., Vol. 6, No. 6, December 1984, pp. 1053-1057. (C) Printed in India.

\title{
Spin wave relaxation processes in polycrystalline yttrium iron garnets
}

\author{
S N BHATIA, P B JOSHI and C M SRIVASTAVA \\ Department of Physics, Indian Institute of Technology, Powai, Bombay 400076, India
}

MS received 7 May 1983

\begin{abstract}
The wavevector and temperature dependence of the spinwave linewidth in yttrium iron garnets has been studied for polycrystalline samples of average grain diameter $12.8 \mu \mathrm{m}$ and has been compared with the calculated relaxation times of basic three-magnon confluence and four-magnon scattering processes.
\end{abstract}

Keywords. Relaxation; spin-wave line-widths; yttrium iron garnets

\section{Introduction}

The nature of the intrinsic relaxation processes which determine the dissipative characteristics of magnetically ordered materials is well understood for the crystalline state (Gurevich and Anisimov 1975). The uniform precession mode which is created in the parallel pumping experiment loses its energy into the degenerate magnon modes. The small wavevector $(k)$ magnons thus generated relax by the three-magnon confluence process ( $3 c$ ) while the larger $k$ magnons, $4 \times 10^{5}<k<9 \times 10^{5} \mathrm{~cm}^{-1}$, attain equilibrium via scattering involving four magnons $(4 \mathrm{sc})$. The splitting process requiring magnons of high frequencies is therefore restricted to $k>10^{6} \mathrm{~cm}^{-1}$. The observed temperature and $k$ dependences of these processes have been established to be in accord with the theoretical results (Sparks 1967; Vaks et al 1968; Gurevich and Anisimov 1975). Such an understanding is lacking for the polycrystalline materials. Vrehan's (1966) transit time model has been used successfully by Patton (1970) and Scotter (1972) to explain the observed relaxation time for the $k \rightarrow 0$ limit in large grains but the model fails for finite $k$ and small grain samples. An empirical relation based on the predictions of transit time model for the wavevector dependence of the spin-wave linewidth, obeyed by samples of grain size 1-12 $\mu \mathrm{m}$ has been arrived at by Prakash and Srivastava (1980). However no attempt appears to have been made to correlate such measurements with the fundamental processes involving the magnons. We have measured the spin-wave linewidths of polycrystalline YIG as functions of the wavevector and temperature for samples of various grain sizes and analysed them in terms of the basic scattering processes of the magnons.

\section{Experimental}

Polycrystalline YIG samples (densities $>99 \%$ average grain diameter $0.9-12.8 \mu \mathrm{m}$ ) were prepared using the normal sintering and hot pressing techniques.

A high power microwave bench giving pulses of $4 \mu \mathrm{sec}$ duration, $80 \mathrm{~kW}$ peak power 
with a repetition frequency of 50 pulses/sec and operating at microwave frequency $9.391 \mathrm{GHz}$ was used to study the parallel pumping instability threshold $\left(h_{c}\right)$ in these samples. A reflection-type rectangular cavity operating in $\mathrm{TE}_{104}$ mode was used and the sample was mounted at its $3 \lambda \mathrm{g} / 2$ position in a vacuum-jacketed glass tube. Liquid nitrogen vapour was blown through this tube to regulate the sample temperature between 120 and $300 \mathrm{~K}$.

\section{Results}

The instability threshold field $\left(h_{c}\right)$ has been observed as a function of the applied magnetic field $H_{0}$. The spin-wave linewidth $\left(\Delta H_{k}\right)$ is calculated from $h_{c}$ through the relation

$$
h_{c}=\left(\omega_{k} / \omega_{m}\right) /\left(\Delta H_{k} / \sin ^{2} \theta_{k}\right),
$$

where $\omega_{k}$ is the operating frequency, $\omega_{m}=4 \pi \gamma M$ and $\theta_{k}$ is the angle between the direction of propagation of the spin-waves and $H_{0}$. The $h_{c}$ vs $H_{0}$ curve was used to calculate $\Delta H_{k}$ up to $\left(h_{c}\right)_{\min }$, the corresponding $k$ value being obtained from the standard dispersion relations. $\Delta H_{k}$ vs $k$ curves for the grain size $12.8 \mu \mathrm{m}$ are shown in figure 1. Temperature is displayed as a parameter on these curves.

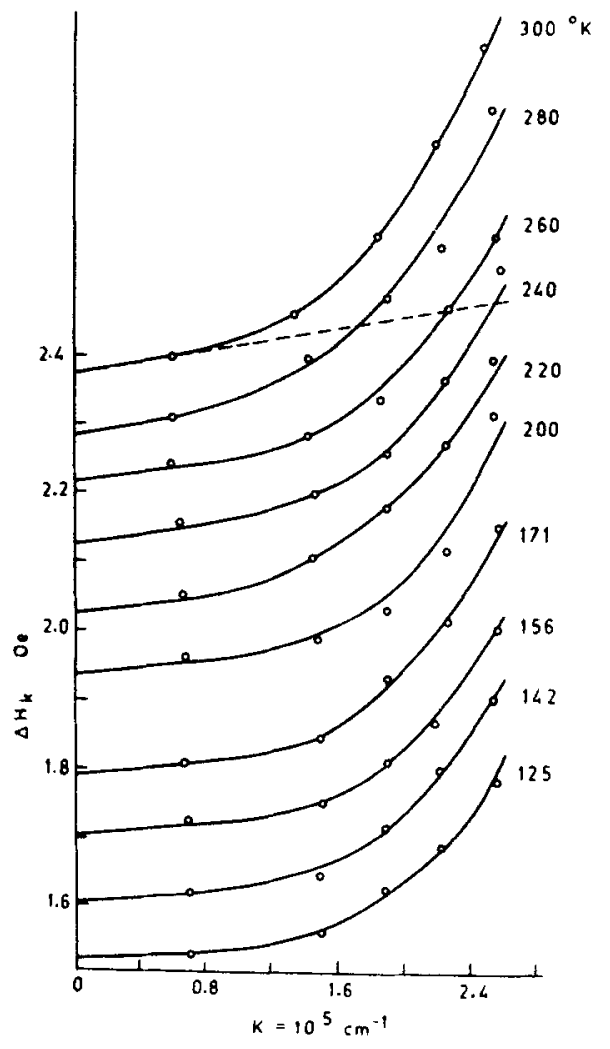

Figure 1. Wayevector dependence of the spinwave line-width for polycrystalline YIG. 
The striking feature of these curves is the nonlinear dependence of $\Delta H_{k}$ on $k$ for all temperatures. This is in contrast to the linear dependence reported earlier (Patton 1970; Prakash and Srivastava 1980) and is attributed to the rather large range of $k$ used in the present graphs. Over the limited range of $1.5 \times 10^{5}<k<3 \times 10^{5} \mathrm{~cm}^{-1}$ used in earlier studies, the present data also shows an almost linear dependence of $\Delta H_{k}$ on $k$. A similar linear dependence was also observed by White et al (1979) between 88 and $368 \mathrm{~K}$. Their most dense sample appears to have lesser $4 \pi M_{s}$ and therefore more porosity than ours. Pores could be the dominant scattering centres (Scotter 1972).

The initial portion of each curve however appears to be a straight line up to a certain value of the wavevector $k_{\min }$, after which the nonlinearities begin. $k_{\min }$ appears to decrease with increasing temperature. This initial portion of each graph was fitted to the equation,

$$
\Delta H_{k}^{\prime}=\Delta H_{k \rightarrow 0}(T)+a(T) k .
$$

After substracting $\Delta H_{k}^{\prime}$ from the observed $\Delta H_{k}$ for $k>k_{\min }$, the difference $\Delta H_{k}-\Delta H_{k}^{\prime}$ was fitted to

$$
\Delta H_{k}-\Delta H_{k}^{\prime}=b(T) k^{2}
$$

for each temperature. The observed variations of $a(T)$ and $b(T)$ with temperature are shown in figure 2. Also shown in this figure is the number of thermal magnons excited $n(T)$ which is linear with $T$ as the energy of these magnons

$$
\hbar w_{k} \approx \gamma H_{0}+D k^{2} \approx \gamma H_{0} \approx 1, k_{\beta} \ll k_{\beta} T .
$$

The increase in $a(T)$ and $b(T)$ with temperature can be attributed to the larger number of magnons present at higher temperatures. Two processes viz the three-magnon confluence and the four-magnon scattering brought on by the dipolar forces, show respectively a linear and a quadratic dependence on both $k$ and $T$. Their respective contributions to $\Delta H_{k}$ for single crystals are

$$
\begin{aligned}
\left(\Delta H_{k}\right)_{3 c} & =\frac{\pi M \gamma k_{B}}{2 D \omega_{k}} T k=C_{3 c} T k \\
\left(\Delta H_{k}\right)_{4 s c} & =\frac{1}{3 \pi} \frac{\omega_{k}}{\gamma} \frac{k_{\beta}^{2} T^{2}}{(4 \pi M D)^{2}}\left(\ln \frac{\hbar w_{k}}{k_{\beta} T}\right)^{2} k^{2} \\
& =C_{4 s c} T^{2} k^{2} .
\end{aligned}
$$

These expressions are valid for magnons with predominant Zeeman energy $\left(D k^{2} \ll \gamma H_{0}\right)$ and at high temperatures, $\left(\hbar \omega_{k} \ll k_{\beta} T\right)$. As mentioned above both these conditions are valid here.

We notice from figure 2 that the coefficients $a(T)$ and $b(T)$ are roughly linearly and quadratically proportional to $T$ suggesting that (1) and (2) could be representing the $3 c$ and $4 s c$ processes and consequently the proportionalities.

$$
\begin{aligned}
& a(T) \propto C_{3 c}, \\
& \text { and } \quad b(T) \propto C_{4 s c} .
\end{aligned}
$$

However (3) and (4) which are valid for single crystals cannot be used directly here for polycrystalline materials as their contributions to $\Delta H_{k}$ are an order of magnitude smaller than the experimentally observed values at all temperatures. Thus for 

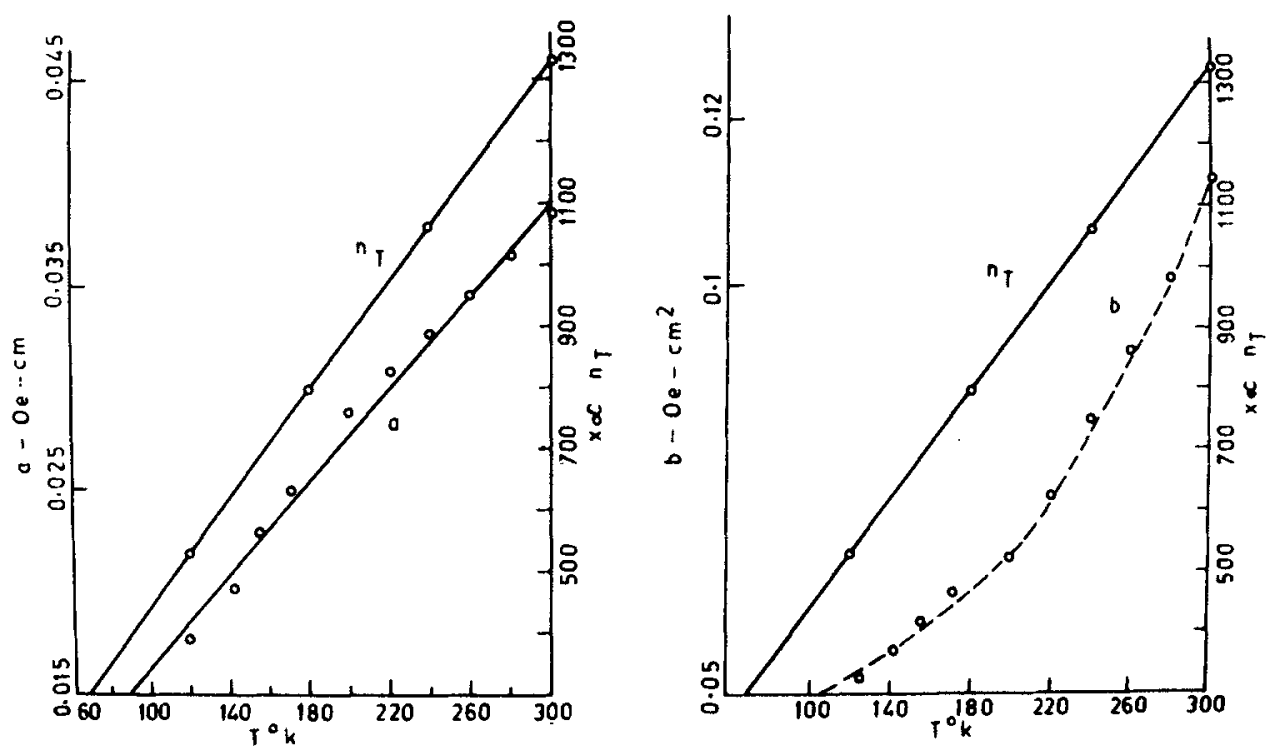

Figure 2. Temperature variation of the coefficients $a$ and $b . n(T)$ is proportional to the number of thermal magnons excited at these temperatures.

$k=2 \times 10^{5} \mathrm{~cm}^{-1}$ experimental $\Delta H_{k}$ values are $0 \cdot 2$ and $0.1 \mathrm{Oe}$ at 300 and $120 \mathrm{~K}$ respectively as against 0.02 and $0.004 \mathrm{Oe}$ calculated for the $4 s c$ processes. Thus it appears that in changing over from the single crystal to the polycrystalline materials, only the form of these expressions is preserved.

$\Delta H_{k \rightarrow 0}$ obtained from figure 1 is plotted against $T$ in figure 3. Again a linear dependence on $T$ is indicated with the data at the lowest temperatures showing some deviations from this line. This is again contrary to the observations of White et al (1979) who report an essentially temperature-independent $\Delta H_{k}$, and is also contradictory to the transit time model. This dissipation parameter contains contributions which may depend upon $k$ but do not vanish as $k \rightarrow 0$. In the present case, $3 c$ and $4 s c$ processes do not contribute to $\Delta H_{k \rightarrow 0}$. Major contribution to $\Delta H_{k \rightarrow 0}$ is stipulated to come from the Kasuya-LeCraw magnon-phonon processes (Sparks 1964) which show a similar temperature dependence. Here again magnitude-wise the observed $\Delta H_{k \rightarrow 0}$ is an order of magnitude larger than that predicted for single crystals (Sparks 1964). However more data at temperatures below $120 \mathrm{~K}$ are required to justify this association.

\section{Conclusion}

For the sample with average grain size $12.8 \mu \mathrm{m}$ the dissipation processes occurring appear to be identical to those in single crystals with two major differences, (i) the minimum $k$ value at which the $4 s c$ processes start contribution is significantly smaller here $\left(k_{\min }<1.2 \times 10^{5} \mathrm{~cm}^{-1}\right.$ at $\left.300 \mathrm{~K}\right)$ than in single crystals (typically $2 \times 10^{5} \mathrm{~cm}^{-1}$ ) and (ii) the magnitudes of the contributions of the respective processes are an order of magnitude greater here than in single crystals. This increased scattering of magnons could be due to the grain boundaries present in the polycrystalline materials. This 


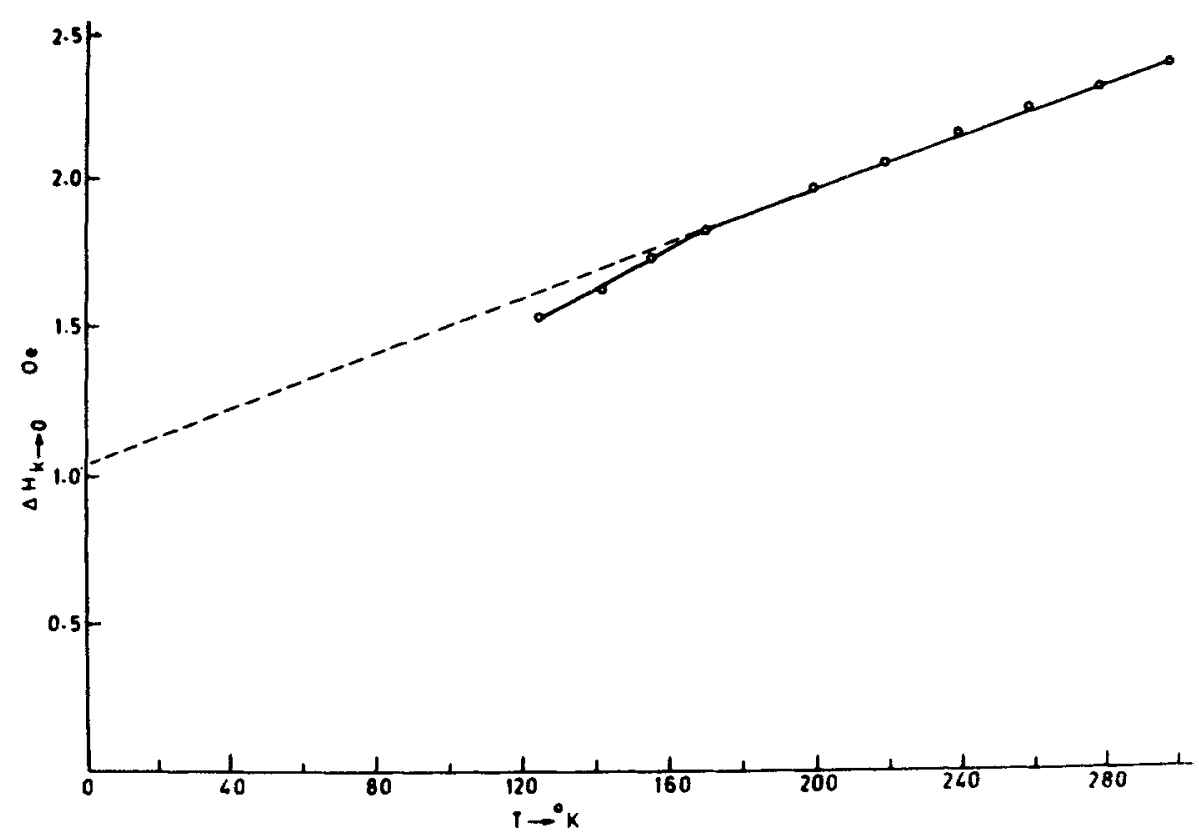

Figure 3. Temperature dependence of the spin-wave linewidth $\Delta H_{k}$ for $k \rightarrow 0$. Note the deviations from linearity at the lower temperatures.

scattering should be more intense in smaller grain samples where there are more boundaries which is in qualitative agreement with the previous observations of Prakash and Srivastava (1980). The details of this mechanism are being investigated.

\section{References}

Gurevich A G and Anisimov A N 1975 Sov. Phys. 41336

Patton C E 1970 J. Appl. Phys. 411637

Prakash O and Srivastava C M 1980 Bull. Mater. Sci. 2181

Scotter D G 1972 J. Phys. D5 L93

Sparks M 1967 Phys. Rev. 160364

Sparks M 1964 Ferromagnetic relaxation theory (New York: McGraw-Hill)

Vaks V G, Larkin A I and Pikin S A 1968 Sov. Phys. JETP 26647

Vrehans Q H F 1966 J. Appl. Phys. 401849

White G, Patton C E and Edmondson 1979 J. Appl. Phys. 502118 